\title{
La competencia creativa entre el alumnado de los grados en Publicidad y Relaciones Públicas en España y su adecuación a las demandas del sector profesional
}

The creative competition between the students of the degrees in Advertising and Public Relations in Spain and its adaptation to the demands of the professional sector

A concorrência criativa entre os alunos dos graus Publicidade $e$ Relaçoes Públicas em Espanha e a sua adequação às exigências do sector profissional

Paloma Sanz-Marcos

Profesora Ayudante Doctor

(Universidad de Cádiz)

http://orcid.org/0000-0002-6103-6993

España
Cristina González-0ñate

Profesora Titular

(Universitat Jaume I)

https://orcid.org/0000-0003-3509-0117

España

\author{
Gloria Jiménez-Marín \\ Profesora Titular \\ (Universidad de Sevilla) \\ https://orcid.org/0000-0003-0252-3975
}

España

Fecha de recepción: 1 de septiembre de 2020

Fecha de revisión: 4 de febrero de 2021

Fecha de aceptación: 13 de mayo de 2021

Fecha de publicación: 1 de julio de 2021

Para citar este artículo: Sanz-Marcos, P., González-Oñate, C. y Jiménez-Marín, G.(2021). La competencia creativa entre el alumnado de los grados en Publicidad y Relaciones Públicas en España y su adecuación a las demandas del sector profesional, Icono 14, 19(2), 66-92. doi: 10.7195/ri14.v19i2.1604 


\section{Resumen}

Este trabajo investiga el desarrollo de la competencia creativa por parte del alumnado durante su formación en el Grado en Publicidad y Relaciones Públicas. Concretamente, se persigue conocer si el estudio e impulso de la creatividad se adecua a las demandas del sector profesional publicitario. Se utiliza una metodología de triple enfoque basada en un análisis de contenido de los planes de estudio, un cuestionario dirigido a alumnos egresados y un panel de expertos formado por académicos pertenecientes al Grado en Publicidad y Relaciones Públicas. Los resultados indican que, si bien la formación de estos graduados parece adecuada en términos generales, la formación recibida en torno a la creatividad parece insuficiente para dar respuesta a las demandas del mercado laboral. Algunos de los factores que explican estas tendencias son el exceso de integración de competencias digitales como consecuencia de la renovación del Grado o la imposibilidad del profesorado para implementar metodologías creativas en el aula debido a la complicada conciliación entre las exigencias académicas y la investigación a las que se ven sometidos. Se recomienda una transformación de los planes de estudio universitarios del Grado en Publicidad y Relaciones Públicas para atender a las demandas laborales vinculadas al ejercicio de la creatividad publicitaria en España.

Palabras clave: Creatividad; Publicidad; Relaciones Públicas; Competencias; Grado; Sector profesional

\section{Abstract}

This paper intends to carry out an investigation about the development of the creative competence during the formation on the university degree of Advertising and Public Relations. Specifically, it seeks to find out if the study and promotion of creativity is adapted to the demands of the professional advertising sector. A triple-approach methodology was carried out based on an analysis of the content of the study plans, a questionnaire for graduate students and a panel of experts made up of academics belonging to the degree of Advertising and Public Relations. The results indicate that, although the training of these graduates seems adequate in general terms, the training received on creativity seems insufficient to respond to the demands of the market. Some of the factors that explain these trends are the 
excessive integration of digital competences due to the renewal of the degree or the impossibility of the academics to implement creative methodologies in the classroom due to the complicated reconciliation between the professional and academic demands they are subjected to. That is why a transformation of the university study plans of the Degree in Advertising and Public Relations is recommended to meet the labor demands related to the exercise of advertising creativity in Spain.

Keywords: Creativity; Advertising, Public Relations; Competencies; Degree; Professional Sector

\section{Resumo}

Este artigo realiza uma investigação sobre o desenvolvimento da competência criativa dos alunos durante a sua formação no Curso de Licenciatura em Publicidade e Relações Públicas. Especificamente, busca saber se o estudo e o impulso da criatividade estão adaptados às demandas do setor publicitário profissional. Para tal, foi realizada uma metodologia de abordagem tripla baseada na análise de conteúdo dos planos de estudos, um questionário dirigido a alunos de pós-graduação e um painel de especialistas constituído por académicos do Curso de Publicidade e Relações Públicas. que, embora a formação desses egressos pareça adequada em termos gerais, a formação recebida em torno da criatividade parece insuficiente para responder às demandas do mercado de trabalho. Alguns dos fatores que explicam essas tendências são o excesso de integração das competências digitais em consequência da renovação do curso ou a impossibilidade dos professores de implementar metodologias criativas em sala de aula devido a dificil conciliação entre os requisitos profissionais e académicos a que estão sujeitos. É por isso que se recomenda uma transformação dos planos de estudos universitários da Licenciatura em Publicidade e Relações Públicas para responder às demandas laborais relacionadas com o exercício da criatividade publicitária em Espanha.

Palavras chave: Criatividade; Publicidade; Relações públicas; Competências; Grau; Setor profissional 
MONOGRÁFICO

\section{Introducción}

Instaurada en nuestra vida cotidiana, la creatividad es un proceso intelectual ligado a la originalidad y al ingenio cuya finalidad es estimular la imaginación creadora para producir ideas válidas ante un problema concreto (Costa, 2015). Esta capacidad es considerada como uno de los rasgos de personalidad más valorados por las empresas a la hora de incorporar nuevos empleados a cualquier ámbito profesional (McKinsey, 2018). Dando respuesta a las exigencias del mercado laboral, en el marco universitario existen diversos Grados que incluyen dentro de sus planes de estudio el fomento de la creatividad de manera reglada. Esta investigación analiza el estudio de la creatividad en la academia con objeto de conocer si esta se adecua a las demandas del sector profesional publicitario. Se propone un análisis del Grado en Publicidad y Relaciones Públicas, estudios cuyo perfil profesional se encamina hacia al desarrollo de la creatividad en el entorno laboral (ANECA, 2005). Se han ido avanzando las carencias de los Grados académicos (Agüero et al., 2019; Farfán y Corredor, 2010), de los estudios de máster (Matilla, Cuenca-Fontbona y Compte-Pujol, 2018) o de los de doctorado (Sánchez Pozo, 2018) con respecto a las demandas profesionales. No obstante, apenas existen estudios que destaquen la importancia del desarrollo de capacidades concretas, en este caso la creatividad, en relación con la expectativa profesional, exceptuando el trabajo de Castelló-Martínez (2020), quien profundiza en perfiles similares como el del planner. Las investigaciones mencionadas, aunque abordan las posibles carencias de los planes de estudio de manera general y en relación con la realidad profesional, no inciden en capacidades concretas, como el trabajo creativo y su posterior reflejo en la futura labor profesional.

Existe una extendida percepción de la Academia como una institución desconectada de la realidad profesional que genera un alto grado de desmotivación entre los estudiantes (Alonso et al., 2009). Esta apreciación ha sido una preocupación presente en la renovación del diseño de los títulos académicos que poco a poco tratan de incluir cambios en el diseño de sus planes de estudio (Vázquez Gestal y Fernández Souto, 2012). Sin embargo, estas mejoras parecen apuntar en un sentido único que recoge exclusivamente la transformación digital. La proliferación de nuevas plataformas, junto con la evolución del entorno 2.0, han sido identificadas en los planes de estudio de los Grados españoles de Publicidad y Relaciones Públi- 
cas como las principales premisas que tener en cuenta a la hora de replantear la incorporación de nuevas asignaturas que traten de dar respuesta al nuevo entorno. Estas propuestas parecen obviar ciertas cualidades que se presuponen básicas y necesarias para el desarrollo exitoso del profesional publicitario. Los investigadores Agüero et al. revelan que asignaturas como Pensamiento Creativo - entre otrassiguen siendo capitales en la formación de los futuros profesionales de la comunicación (2019). Si bien este enfoque es necesario dadas las exigencias actuales de la profesión, esta apreciación pone de manifiesto la marcada preocupación por renovar los Grados teniendo en cuenta únicamente el aspecto tecnológico del mercado, relegando a un segundo plano el desarrollo de destrezas como la creatividad, además del deseo de muchos estudiantes que se decantan por el trabajo profesional creativo (Cuenca, Compte y Matilla, 2017; Peinado y Fernández, 2011). Por esta razón, esta investigación acerca la realidad profesional y académica, así como a sus estudiantes y profesionales en activo, con objeto de contribuir al avance y posible mejora del rendimiento y competitividad académicos.

\subsection{La enseñanza de la creatividad en las aulas universitarias españolas}

La tradicional creencia acerca de que la creatividad es una propiedad mágica que caracteriza a ciertas personas privilegiadas es una concepción que ha sido ampliamente superada por el trabajo de investigadores y expertos que han consensuado que, en efecto, se trata de una aptitud que puede ser estimulada (Selva Ruíz y Domínguez Liñán, 2018: p. 372). Y es que, a pesar de que la creatividad es un proceso que puede ser complejo ya que emplea capacidades como la imaginación (Villamizar, 2012), es posible orientarla y trabajarla hasta ser aprendida (Robinson, 2009). Uno de los objetivos que plantea esta habilidad es buscar nuevas soluciones a problemas ya existentes. En el caso que nos ocupa su dificultad estriba en que no se trata de una "creación libre, lúdica, gratuita", por el contrario "está supeditada a un brief o pliego de condiciones técnicas, y éste a su vez lo está a unos objetivos" (Costa, 2015: p. 18).

Este reto, unido a otras limitaciones como el tiempo, son los desafíos que caracterizan el día a día del trabajo desarrollado por las agencias. Investigaciones 
como las de Selva Ruíz y Domínguez Liñán (2018) manifiestan que las agencias de publicidad emplean rutinariamente diversas técnicas que persiguen dinamizar el pensamiento creativo para la generación de ideas de calidad ante la creación de una determinada campaña. Siguiendo a Vázquez Gestal (2011), la creatividad es un instrumento esencial en el ámbito publicitario que ayuda a resolver los problemas de comunicación que los anunciantes tienen con sus consumidores. Es por ello por lo que se trata de una de las competencias básicas que todo futuro publicitario habría de desarrollar en su formación.

En el ámbito universitario, los Grados de comunicación y, de manera concreta, el Grado en Publicidad y Relaciones Públicas, se presentan como la opción natural para la formación de futuros creativos publicitarios (ANECA, 2015). Sin embargo, estudios previos plantean que el diseño de estas titulaciones no responde directamente a las demandas del sector (Castelló-Martínez, 2020). Agüero et al. (2019) afirman que los profesionales han detectado ciertas carencias estrechamente vinculadas con la formación del pensamiento creativo cuando indican que se trata de una práctica que, estando íntimamente relacionada con su aplicación práctica en el mundo profesional, se encuentra prácticamente olvidada dentro del modelo educativo actual. Farfán y Corredor encuentran que estas carencias pueden verse afectadas por "la falta de experiencia profesional o de contacto con la realidad de los profesores de la asignatura de creatividad" (2012: p. 108), ampliando el problema no solo al diseño de los planes de estudio, sino a la formación del profesorado. Otros autores han debatido acerca de la dificultad que el profesorado universitario tiene para conciliar las exigencias docentes con aquellas relativas a la investigación. Sáenz (1990) señala que la falta de apoyo institucional, la formación específica y la ausencia de cursos de perfeccionamiento, son factores que dificultan la versatilidad de estos perfiles. En efecto, la formación del personal docente universitario es fundamental a la hora de afrontar este ajuste con el entorno profesional (Ruíz y Martín, 2005).

Estas propuestas han ido calando de forma progresiva en la innovación docente universitaria. Las exigencias que demandaban un cambio en el desfase producido por una enseñanza basada exclusivamente en los contenidos y la enseñanza magistral (Martínez et al., 2013), han evolucionado hacia propues- 
La competencia creativa entre el alumnado de los grados en Publicidad... | 72 MONOGRÁFICO

tas innovadoras que proponen una perspectiva en la que el docente ocupa un lugar más cercano a la figura del facilitador del aprendizaje y mediador (Bain, 2004; 2011; Imbernón y Guerrero, 2018; Román-San-Miguel, 2014). No obstante, siguiendo la premisa de atender a aquellas reivindicaciones que buscan una formación lo más cercana posible a la realidad profesional (Torres, 2008) la mayoría de las transformaciones responden a la tarea de reducir la innovación exclusivamente a la utilización de dispositivos tecnológicos en las aulas Fueyo, Rodríguez-Hoyos y Linares (2015), poniendo de manifiesto que la brecha entre lo académico y lo profesional es todavía un reto para la Academia (Vázquez Gestal y Fernández Souto, 2012).

\subsection{El desarrollo creativo en el ámbito profesional publicitario.}

Integrada en el sector económico de los servicios, la actividad publicitaria se ha convertido en una "actividad transversal de profundas consecuencias para la vida política, económica y social de España" (Alameda-García, Fernández-Blanco y Benavides-Delgado, 2013). Así lo evidencia el estudio elaborado por el Observatorio de la Publicidad en España (2019), que refleja que el total de la inversión publicitaria se eleva a 12.836 millones de euros, una cifra que crece exponencialmente desde 2014. Este dato representa un 3,5\% del volumen de todo el sector servicios en España, indicando que se trata de un sector de la economía. Estos datos son ampliados con los presupuestos que las empresas destinan en comunicación. Según Scopen (2019), estos presupuestos han crecido un 5\% desde 2016.

En definitiva, estos estudios ponen de manifiesto la resistencia y capacidad de renovación de un sector que, a pesar de las crisis, ha conseguido mantenerse ante los constantes cambios y exigencias. Parte de este triunfo se debe a la incuestionable labor de sus profesionales. En el caso concreto de las agencias de publicidad, sus creativos se han visto imbuidos en una evolución forzada debido a la rapidez con la que se han ido sucediendo los cambios en la transformación de medios (González-0ñate, 2019). Estos desafíos plantean importantes retos tanto a nivel estratégico como a nivel organizativo para las agencias (LópezFont, 2005). Por su parte, Vázquez Gestal y Fernández Souto manifiestan que los propios expertos valoran positivamente "la necesidad de saber contar historias, 
de buscar ideas, de producir resultados novedosos y originales" en los profesionales con los que trabajar (2012: pp. 896-897). En efecto, la creatividad es una herramienta estratégica en todos los momentos del proceso publicitario, ya sea durante la fase estratégica, la concepción del mensaje o la materialización de la idea (Solanas y Sabaté, 2011).

\section{Objetivos y Metodología de la investigación}

Partiendo de las investigaciones anteriormente propuestas, el presente estudio trata de dar respuesta a la siguiente hipótesis: las actuales demandas laborales vinculadas al ejercicio de la creatividad publicitaria en España reclaman una transformación de los planes de estudio universitarios del Grado en Publicidad y Relaciones Públicas. De la hipótesis se desprende como objetivo principal:

1. Identificar y analizar los planes de estudio del Grado en Publicidad y Relaciones Públicas en España atendiendo a aquellas asignaturas específicas de creatividad con la finalidad de ver si la organización de estos planes se adecua a las demandas del sector profesional publicitario.

Acompañando a este objetivo principal, se definen los siguientes objetivos secundarios:

- 0.1.1. Examinar el ajuste de los planes de estudio a los perfiles creativos que se demandan en el campo profesional.

- 0.1.2. Evaluar la adecuación de la formación recibida sobre creatividad en el Grado en Publicidad y Relaciones Públicas por los alumnos egresados con respecto a su desempeño profesional.

Como se observa en la tabla 1, para la consecución de los objetivos propuestos de esta investigación, se emplea una metodología cualitativa y cuantitativa apoyada en diferentes técnicas y fases: 
La competencia creativa entre el alumnado de los grados en Publicidad... | 74

MONOGRÁFICO

\begin{tabular}{|c|c|c|c|}
\hline & Técnica empleada & Perfil investigado & $\begin{array}{c}\text { Correspondencia } \\
\text { con objetivos }\end{array}$ \\
\hline FASE 1 & $\begin{array}{c}\text { Análisis de contenido } \\
\text { (Krippendorf, 2004) }\end{array}$ & $\begin{array}{c}\text { Plan de estudios del Grado en Publi- } \\
\text { cidad y Relaciones Públicas }\end{array}$ & Objetivo 1 \\
\hline FASE 2 & $\begin{array}{c}\text { Cuestionario (Martínez } \\
\text { 0lmo, 2002) }\end{array}$ & $\begin{array}{c}\text { Alumnos egresados y Personal do- } \\
\text { cente e investigador }\end{array}$ & 0bjetivo 1.2. \\
\hline FASE 3 & $\begin{array}{c}\text { Panel de expertos } \\
\text { (Linstone y Turoff 2002) }\end{array}$ & Creativos profesionales & 0bjetivo 1.1. \\
\hline
\end{tabular}

Tabla 1: Fases de la metodología de investigación empleada. Fuente: Elaboración propia.

\section{Fase metodológica 1}

La primera fase se inicia con el análisis de los planes de estudio de los Grados de Publicidad y Relaciones Públicas. Para ello, se lleva a cabo una exhaustiva revisión documental de todos los planes de estudio de instituciones universitarias, públicas y privadas, que imparten estos estudios en España. Esta búsqueda contempla la totalidad de Grados encontrados a través del Buscador de Títulos Universitarios facilitado en la web de ANECA. Se revisan los planes de estudio de 53 instituciones universitarias nacionales: 1 en Alicante, 6 en Barcelona, 1 en Cádiz, 1 en Cantabria, 1 en Castellón de la Plana, 1 en Girona, 1 en Islas Baleares, 6 en Madrid, 1 en Málaga, 2 en Murcia, 1 en Navarra, 1 en Pontevedra, 1 en Sevilla, 2 en Valencia, 1 en Valladolid, y 1 en Vizcaya. La revisión documental se lleva a cabo durante el mes de marzo de 2020 y acota los planes de estudio previstos para el año académico $2019 / 2020$.

En cuanto a la concreción de los contenidos analizados, se ha llevado a cabo un análisis de contenido (Krippendorf, 2004) a través de una plantilla basada en el análisis previo sobre las asignaturas de comunicación en los MBA españoles realizado por Matilla, Cuenca-FontBona y Compte-Pujol (2018). Esta plantilla se aplica a los programas presentes en las diversas webs de los centros identificados y comprende las siguientes variables: provincia en la que está ubicado cada centro; nombre de la universidad y carácter (privada o pública), denominación de las asignaturas relacionadas con la creatividad; número y porcentaje de créditos ECTS asignados a cada asignatura; tipo de la asignatura (obligatorias u optativas); y curso en el que se imparte. 


\section{MONOGRÁFICO}

\section{Fase metodológica 2}

Para evaluar la adecuación de la formación recibida sobre creatividad en el Grado en Publicidad y Relaciones Públicas por los alumnos egresados con respecto a su desempeño profesional (objetivo 1.2), se ha llevado a cabo un cuestionario multirrespuesta (Martín González, 2010) a alumnos egresados del Grado en Publicidad y Relaciones Públicas. La encuesta fue aplicada a través de Internet mediante la plataforma Google Forms; para la explotación de datos se utilizó el software específico SPSS. Los cuestionarios se llevaron a cabo entre los días 15 de noviembre de 2019 y 15 de enero de 2020 y se cumplimentaron un total de 245 cuestionarios de los cuales 196 fueron válidos. El cuestionario de ocho preguntas cerradas multirrespuesta se estructuró a partir de cinco bloques temáticos: Situación laboral actual del egresado, competencias desarrolladas en su actividad profesional actual, estimación de la falta de formación a la incorporación al mundo laboral, valoración de las metodologías recibidas durante la formación en materia de creatividad y valoración del desarrollo de la creatividad durante la formación universitaria.

La muestra se seleccionó bajo los siguientes criterios:

- Egresados incorporados al mercado laboral como empleados en activo dentro del sector publicitario.

- Egresados con diferentes perfiles de desempeño dentro de la actividad publicitaria.

Dado que el profesorado encarna un papel primordial tanto en la percepción como en la formación del alumnado, se ha considerado pertinente contar con la opinión de 36 docentes con dedicación completa en el Grado en Publicidad y Relaciones Públicas. Al igual que en el cuestionario dirigido a los egresados, la encuesta fue realizada a través de la plataforma Google Forms y posteriormente explotada mediante software SPSS. En este caso el trabajo de campo se realizó entre el 20 de enero y 20 de marzo de 2020. En cuanto a la selección de los encuestados se fijó el siguiente criterio: personal docente e investigador contratado a tiempo completo con docencia en el Grado en Publicidad y Relaciones Públicas que hubiera impartido alguna asignatura dedicada al desarrollo de la creatividad publicitaria. 
La competencia creativa entre el alumnado de los grados en Publicidad... | 76 MONOGRÁFICO

El cuestionario, compuesto por 10 preguntas cerradas de respuesta múltiple, considera cuatro bloques temáticos relacionados con las premisas destacadas por los estudios previamente comentados:

- Bloque 1: dedicado a conocer el perfil del encuestado.

- Bloque 2: destinado a conocer la valoración de la metodología de enseñanza empleada en el aula (Ruíz y Martín, 2005).

- Bloque 3: cuyo objetivo trata de recoger la valoración de los planes de estudio del Grado en Publicidad y Relaciones Públicas en relación con las necesidades del mercado laboral actual.

- Bloque 4: Valoración del apoyo institucional en cuanto a recursos para el desarrollo de la materia (Sáenz, 1990) y formación del profesorado (Farfán y Corredor, 2012).

\section{Fase metodológica 3}

Una vez finalizadas las primeras fases, se inicia la dedicada a la celebración de un panel de expertos (Linstone y Turoff, 2002), que busca analizar la complejidad y particularidades del trabajo creativo con objeto de examinar la adecuación de los planes de estudios a la realidad profesional demandada (objetivo 1.1). Los expertos en cuestión proceden de las siguientes agencias publicitarias: E1 (Family Different), E2 (Officer \& Gentelman), E3 (Singular One), E4 (Dakota \& Durango), E5 (Miwerta), E6 (La caseta de Juan León), E7 (Grupo Mundo), E8 (McCann Erickson). Los criterios de selección de los expertos han pivotado en torno a tres conceptos fundamentales. En primer lugar, se ha buscado la afinidad de los expertos con el objeto de estudio, en este caso, la creatividad publicitaria; en segundo lugar, se ha valorado el prestigio y/o reconocimiento obtenido a nivel nacional en el ámbito profesional de los miembros y en relación con el objeto de estudio. Finalmente, se ha buscado la constitución de un grupo que, a pesar de mantenerse unido por un hilo conductor previamente definido, presentara perfiles bastante heterogéneos para enriquecer el discurso y el resultado final.

El panel fue desarrollado de forma electrónica; se envió a los expertos una invitación por correo electrónico presentándoles la investigación junto a un informe y 


\section{MONOGRÁFICO}

formulario de preguntas abiertas. Las preguntas se dirigen a reforzar y justificar los resultados del análisis de contenido y cuestionarios previos proponiendo así una intervención directa de los participantes. Con objeto de trasladar los resultados obtenidos en las fases previas, se elaboró un informe con la explotación de los resultados obtenidos para poder discutir durante la celebración del panel de expertos junto con las preguntas. Las respuestas extraídas fueron codificadas por dimensiones en categorías verbales y se transcribieron por áreas temáticas los datos de las entrevistas. Estos resultados se presentan por categorías temáticas atendiendo así a las cuestiones que analizamos. La aportación de los expertos figura identificada entre comillas y se atribuye a un código asignado previamente a cada uno de ellos, que aparece entre paréntesis, donde la $E$ hace alusión al término experto, seguida del número que le corresponde según el orden secuencial atribuido.

En definitiva, el desarrollo de esta metodología en tres fases trata de dar respuesta a los objetivos planteados a través de la implicación de cada uno de los actores directamente implicados en la actividad académica publicitaria, proporcionando así una visión múltiple.

\section{Resultados}

Con objeto de analizar y extraer los datos que nos conducirán a las conclusiones, presentamos los resultados atendiendo al orden de las distintas fases metodológicas.

\section{Resultados Fase 1}

Del análisis de los planes de estudio de los Grados de Publicidad y Relaciones Públicas de las 38 instituciones estudiadas se identificaron un total de 53 asignaturas dedicadas a la creatividad.

\begin{tabular}{|l|c|c|c|c|c|c|c|c|}
\hline Provincia & Universidad & Caráter & Denominación & Etcs & $\%$ Etcs & Tipo & Curso & \\
\hline Alicante & U. de Alicante & Pública & $\begin{array}{c}\text { Fundamentos de la } \\
\text { Creatividad }\end{array}$ & 6 & $2,50 \%$ & OB & $2^{\circ}$ & \\
\hline Alicante & U. de Alicante & Pública & $\begin{array}{c}\text { Estrategia Creativa } \\
\text { y Conceptualiza- } \\
\text { ción }\end{array}$ & 6 & $2,50 \%$ & $0 \mathrm{~B}$ & $3^{\circ}$ & \\
\hline
\end{tabular}


La competencia creativa entre el alumnado de los grados en Publicidad... | 78

MONOGRÁFICO

\begin{tabular}{|c|c|c|c|c|c|c|c|c|}
\hline Provincia & Universidad & Caráter & Denominación & Etcs & \% Etcs & Tipo & Curso & \\
\hline Barcelona & $\begin{array}{l}\text { Autónoma de } \\
\text { Barcelona }\end{array}$ & Pública & $\begin{array}{c}\text { Creatividad Publi- } \\
\text { citaria }\end{array}$ & 12 & $5,00 \%$ & OB & $2^{\circ}$ & \\
\hline Barcelona & $\begin{array}{l}\text { Autónoma de } \\
\text { Barcelona }\end{array}$ & Pública & $\begin{array}{c}\text { Procesos y } \\
\text { Ténicas Creativas }\end{array}$ & 6 & $2,50 \%$ & OB & $3^{\circ}$ & \\
\hline Barcelona & $\begin{array}{l}\text { Autónoma de } \\
\text { Barcelona }\end{array}$ & Pública & $\begin{array}{l}\text { Creatividad } \\
\text { Audiovisual }\end{array}$ & 6 & $2,50 \%$ & OT & $4^{0}$ & $\begin{array}{l}\text { Mención en } \\
\text { Dirección } \\
\text { Creativa en } \\
\text { Publicidad y } \\
\text { Relaciones } \\
\text { Públicas }\end{array}$ \\
\hline Barcelona & $\begin{array}{l}\text { Autónoma de } \\
\text { Barcelona }\end{array}$ & Pública & Creatividad Gráfica & 3 & $1,25 \%$ & OT & $4^{0}$ & $\begin{array}{l}\text { Mención en } \\
\text { Dirección } \\
\text { Creativa en } \\
\text { Publicidad y } \\
\text { Relaciones } \\
\text { Públicas }\end{array}$ \\
\hline Barcelona & $\begin{array}{l}\text { Autónoma de } \\
\text { Barcelona }\end{array}$ & Pública & Book Creativo & 6 & $2,50 \%$ & OT & $4^{0}$ & $\begin{array}{l}\text { Mención en } \\
\text { Dirección } \\
\text { Creativa en } \\
\text { Publicidad y } \\
\text { Relaciones } \\
\text { Públicas }\end{array}$ \\
\hline Barcelona & Pompeu Fabra & Pública & $\begin{array}{c}\text { Teorías y Técnicas } \\
\text { de la Ideación } \\
\text { Publicitaria }\end{array}$ & 6 & $2,50 \%$ & B & $2^{\circ}$ & \\
\hline Barcelona & Pompeu Fabra & Pública & $\begin{array}{l}\text { Estrategia y } \\
\text { Creatividad }\end{array}$ & 4 & $1,67 \%$ & $\mathrm{OP}$ & $4^{\circ}$ & $\begin{array}{l}\text { Itinerario } \\
\text { Creatividad } \\
\text { Publicitaria }\end{array}$ \\
\hline Barcelona & Pompeu Fabra & Pública & $\begin{array}{l}\text { Creatividad en } \\
\text { Formas Comuni- } \\
\text { cativas de Medios } \\
\text { Interactivos }\end{array}$ & 4 & $1,67 \%$ & $\mathrm{OP}$ & $4^{\circ}$ & $\begin{array}{l}\text { Itinerario } \\
\text { Creatividad } \\
\text { Publicitaria }\end{array}$ \\
\hline Barcelona & Pompeu Fabra & Pública & $\begin{array}{l}\text { Creatividad en } \\
\text { Medios y Soportes } \\
\text { Publicitarios }\end{array}$ & 4 & $1,67 \%$ & $\mathrm{OP}$ & $4^{\circ}$ & $\begin{array}{l}\text { Itinerario } \\
\text { Creatividad } \\
\text { Publicitaria }\end{array}$ \\
\hline Barcelona & Abat Oliba CEU & Privada & Dirección Creativa & 4,5 & $1,88 \%$ & OB & $3^{\circ}$ & \\
\hline Barcelona & $\begin{array}{l}\text { Internacional } \\
\text { de Catalunya }\end{array}$ & Privada & $\begin{array}{l}\text { Creatividad } \\
\text { Publicitaria }\end{array}$ & 5 & $2,08 \%$ & $\mathrm{OP}$ & $3^{\circ}$ & \\
\hline Barcelona & $\begin{array}{l}\text { Vic-Universi- } \\
\text { dad Central de } \\
\text { Catalunya }\end{array}$ & Privada & $\begin{array}{l}\text { Bases of Creative } \\
\text { Thinking and } \\
\text { Innovation }\end{array}$ & 6 & $2,50 \%$ & $\mathrm{OB}$ & $1^{\circ}$ & \\
\hline Barcelona & $\begin{array}{c}\text { Vic-Universi- } \\
\text { dad Central de } \\
\text { Catalunya }\end{array}$ & Privada & $\begin{array}{l}\text { Creatvidad } \\
\text { Aplicada }\end{array}$ & 6 & $2,50 \%$ & $\mathrm{OB}$ & $2^{\circ}$ & \\
\hline
\end{tabular}




\section{MONOGRÁFICO}

\begin{tabular}{|c|c|c|c|c|c|c|c|c|}
\hline Provincia & Universidad & Caráter & Denominación & Etcs & $\%$ Etcs & Tipo & Curso & \\
\hline Barcelona & $\begin{array}{l}\text { Vic-Universi- } \\
\text { dad Central de } \\
\text { Catalunya }\end{array}$ & Privada & $\begin{array}{l}\text { Creatividad en } \\
\text { Internet }\end{array}$ & 3 & $1,25 \%$ & OB & $3^{\circ}$ & \\
\hline Barcelona & $\begin{array}{l}\text { Vic-Universi- } \\
\text { dad Central de } \\
\text { Catalunya }\end{array}$ & Privada & $\begin{array}{l}\text { Creatividad } \\
\text { Publicitaria }\end{array}$ & 3 & $1,25 \%$ & $\mathrm{OP}$ & $4^{0}$ & \\
\hline Barcelona & $\begin{array}{l}\text { U. de Barce- } \\
\text { lona }\end{array}$ & Privada* & $\begin{array}{l}\text { Creativitat } \\
\text { Publicitària }\end{array}$ & 6 & $2,50 \%$ & OB & $2^{\circ}$ & \\
\hline Cádiz & U. de Cádiz & Pública & $\begin{array}{l}\text { Creatividad } \\
\text { Publicitaria }\end{array}$ & 6 & $2,50 \%$ & OB & $3^{\circ}$ & \\
\hline Cantabria & $\begin{array}{l}\text { Europea del } \\
\text { Atlántico }\end{array}$ & Privada & $\begin{array}{l}\text { Creación } \\
\text { Publicitaria }\end{array}$ & 6 & $2,50 \%$ & OB & $3^{\circ}$ & \\
\hline $\begin{array}{l}\text { Castellón } \\
\text { de la Plana }\end{array}$ & $\begin{array}{l}\text { Jaume I de } \\
\text { Castellón }\end{array}$ & Pública & $\begin{array}{c}\text { Creatividad } \\
\text { Publicitaria I }\end{array}$ & 6 & $2,50 \%$ & OB & $3^{\circ}$ & \\
\hline $\begin{array}{l}\text { Castellón } \\
\text { de la Plana }\end{array}$ & $\begin{array}{l}\text { Jaume I de } \\
\text { Castellón }\end{array}$ & Pública & $\begin{array}{c}\text { Creatividad } \\
\text { Publicitaria II }\end{array}$ & 6 & $2,50 \%$ & OB & $3^{\circ}$ & \\
\hline Girona & U. de Girona & Pública & $\begin{array}{c}\text { Procesos y Ténicas } \\
\text { Creativas Publici- } \\
\text { tarias }\end{array}$ & 4 & $1,67 \%$ & OB & $2^{0}$ & \\
\hline Girona & U. de Girona & Pública & $\begin{array}{l}\text { Creatividad } \\
\text { Publicitaria }\end{array}$ & 4 & $1,67 \%$ & OB & $4^{0}$ & \\
\hline Girona & U. de Girona & Pública & $\begin{array}{l}\text { Creación de un } \\
\text { Book }\end{array}$ & 3 & $1,25 \%$ & $\mathrm{OP}$ & $4^{0}$ & \\
\hline $\begin{array}{l}\text { Islas } \\
\text { Baleares }\end{array}$ & $\begin{array}{l}\text { Pontificia de } \\
\text { Comillas }\end{array}$ & Privada & $\begin{array}{c}\text { Creatividad } \\
\text { Publicitaria II }\end{array}$ & 6 & $2,50 \%$ & OB & $4^{0}$ & \\
\hline $\begin{array}{l}\text { Islas } \\
\text { Baleares }\end{array}$ & $\begin{array}{c}\text { Pontificia de } \\
\text { Comillas }\end{array}$ & Privada & $\begin{array}{c}\text { Creatividad } \\
\text { Publicitaria I }\end{array}$ & 6 & $2,50 \%$ & OB & $4^{0}$ & \\
\hline Madrid & San Pablo CEU & Privada & $\begin{array}{l}\text { Pensamiento } \\
\text { Creativo }\end{array}$ & 6 & $2,50 \%$ & OB & $2^{0}$ & \\
\hline Madrid & San Pablo CEU & Privada & $\begin{array}{l}\text { Creatividad } \\
\text { Publicitaria } \\
\end{array}$ & 6 & $2,50 \%$ & OB & $3^{\circ}$ & \\
\hline Madrid & $\begin{array}{l}\text { Rey Juan } \\
\text { Carlos I }\end{array}$ & Privada & $\begin{array}{c}\text { Creatividad en la } \\
\text { Elaboración del } \\
\text { Mensaje Publici- } \\
\text { tario }\end{array}$ & 6 & $2,50 \%$ & OB & $3^{\circ}$ & \\
\hline Madrid & $\begin{array}{l}\text { Complutense } \\
\text { de Madrid }\end{array}$ & Pública & $\begin{array}{c}\text { Creatividad en los } \\
\text { Medios No Conven- } \\
\text { cionales }\end{array}$ & 6 & $2,50 \%$ & $\mathrm{OP}$ & $3^{0}$ & \\
\hline Madrid & $\begin{array}{l}\text { Complutense } \\
\text { de Madrid }\end{array}$ & Pública & $\begin{array}{l}\text { El proceso de } \\
\text { Creación } \\
\text { Publicitaria }\end{array}$ & 6 & $2,50 \%$ & OB & $3^{\circ}$ & \\
\hline Madrid & $\begin{array}{l}\text { Camilo José } \\
\text { Cela }\end{array}$ & Privada & Creatividad & 6 & $2,50 \%$ & OB & $3^{\circ}$ & \\
\hline
\end{tabular}


La competencia creativa entre el alumnado de los grados en Publicidad... | 80

MONOGRÁFICO

\begin{tabular}{|c|c|c|c|c|c|c|c|c|}
\hline Provincia & Universidad & Caráter & Denominación & Etcs & $\%$ Etcs & Tipo & Curso & \\
\hline Madrid & $\begin{array}{c}\text { Camilo José } \\
\text { Cela }\end{array}$ & Privada & $\begin{array}{l}\text { Creatividad } \\
\text { Publicitaria } \\
\end{array}$ & 6 & $2,50 \%$ & OB & $3^{\circ}$ & \\
\hline Madrid & $\begin{array}{c}\text { Antonio de } \\
\text { Nebrija }\end{array}$ & Privada & $\begin{array}{l}\text { Creatividad } \\
\text { Publicitaria } \\
\end{array}$ & 6 & $2,50 \%$ & OB & $3^{\circ}$ & \\
\hline Madrid & $\begin{array}{c}\text { Francisco de } \\
\text { Vitoria } \\
\end{array}$ & Privada & $\begin{array}{c}\text { Taller de } \\
\text { Creatividad I }\end{array}$ & 4,5 & $1,88 \%$ & OB & $1^{0}$ & \\
\hline Madrid & $\begin{array}{c}\text { Francisco de } \\
\text { Vitoria }\end{array}$ & Privada & $\begin{array}{c}\text { Taller de } \\
\text { Creatividad II } \\
\end{array}$ & 4,5 & $1,88 \%$ & OB & $2^{0}$ & \\
\hline Madrid & $\begin{array}{l}\text { Francisco de } \\
\text { Vitoria }\end{array}$ & Privada & Narración Creativa & 6 & $2,50 \%$ & OB & $2^{0}$ & \\
\hline Madrid & $\begin{array}{c}\text { Francisco de } \\
\text { Vitoria }\end{array}$ & Privada & $\begin{array}{c}\text { Expresión Creativa } \\
\text { de la Imagen }\end{array}$ & 6 & $2,50 \%$ & OB & $2^{0}$ & \\
\hline Madrid & $\begin{array}{c}\text { Francisco de } \\
\text { Vitoria }\end{array}$ & Privada & $\begin{array}{c}\text { Expresión Creativa } \\
\text { del Vídeo }\end{array}$ & 6 & $2,50 \%$ & OB & $2^{0}$ & \\
\hline Málaga & U. de Málaga & Pública & $\begin{array}{l}\text { Creatividad } \\
\text { Publicitaria }\end{array}$ & 6 & $2,50 \%$ & OB & $2^{0}$ & \\
\hline Murcia & $\begin{array}{l}\text { Católica San } \\
\text { Antonio }\end{array}$ & Privada & $\begin{array}{l}\text { Creatividad en } \\
\text { Comunicación }\end{array}$ & 6 & $2,50 \%$ & FB & $1^{0}$ & \\
\hline Murcia & U. de Murcia & Pública & $\begin{array}{l}\text { Creatividad } \\
\text { Publicitaria }\end{array}$ & 12 & $5,00 \%$ & OB & $3^{\circ}$ & \\
\hline Navarra & U. de Navarra & Privada & $\begin{array}{l}\text { Creación y Elabora- } \\
\text { ciónd de Mensajes }\end{array}$ & 18 & $7,50 \%$ & OB & $4^{0}$ & \\
\hline Pontevedra & U. de Vigo & Pública & $\begin{array}{l}\text { Creatividad } \\
\text { Publicitaria }\end{array}$ & 6 & $2,50 \%$ & OB & $2^{\circ}$ & \\
\hline Sevilla & U. de Sevilla & Pública & $\begin{array}{l}\text { Creatividad } \\
\text { Publicitaria }\end{array}$ & 6 & $2,50 \%$ & OB & $2^{\circ}$ & \\
\hline Valencia & $\begin{array}{l}\text { Cardenal } \\
\text { Herrera-CEU }\end{array}$ & Privada & $\begin{array}{c}\text { Creatividad para la } \\
\text { Creación publicita- } \\
\text { ria y RRPP }\end{array}$ & 9 & $3,75 \%$ & OB & $3^{\circ}$ & \\
\hline Valencia & $\begin{array}{c}\text { Europea Miguel } \\
\text { de Cervantes }\end{array}$ & Privada & Creatividad & 6 & $2,50 \%$ & OB & $3^{\circ}$ & \\
\hline Valladolid & U. de Valldolid & Pública & $\begin{array}{l}\text { Ideas, Métodos } \\
\text { y Estrategias } \\
\text { Creativas: El plan o } \\
\text { Proyecto Creativo }\end{array}$ & 6 & $2,50 \%$ & OB & $3^{\circ}$ & \\
\hline Valladolid & U. de Valldolid & Pública & Creatividad Online & 3 & $1,25 \%$ & $\mathrm{OP}$ & $4^{0}$ & $\begin{array}{l}\text { Itinerario } \\
\text { Creatividad y } \\
\text { gestión de la } \\
\text { creatividad de } \\
\text { la Comunica- } \\
\text { ción Publici- } \\
\text { taria y de las } \\
\text { Relaciones } \\
\text { Públicas }\end{array}$ \\
\hline
\end{tabular}


81 | Paloma Sanz-Marcos, Cristina González-Oñate y Gloria Jiménez-Marín

MONOGRÁFICO

\begin{tabular}{|l|c|c|c|c|c|c|c|l|}
\hline Provincia & Universidad & Caráter & Denominación & Etcs & $\%$ Etcs & Tipo & Curso & \\
\hline Vizcaya & $\begin{array}{c}\text { U. de País } \\
\text { Vasco }\end{array}$ & Pública & Creatividad & 6 & $2,50 \%$ & OB & $2^{\circ}$ & \\
\hline Vizcaya & $\begin{array}{c}\text { U. de País } \\
\text { Vasco }\end{array}$ & Pública & Creatividad II & 6 & $2,50 \%$ & OP & $3^{\circ}$ & $\begin{array}{l}\text { Mención } \\
\text { Realización } \\
\text { Multimedia en } \\
\text { Publicidad }\end{array}$ \\
\hline
\end{tabular}

Tabla 2: Asignaturas enfocadas a la creatividad en los Grados universitarios de

Publicidad y Relaciones Públicas en España. Fuente: elaboración propia.

*Se indica que, a pesar de que la Universitat de Barcelona es una universidad pública, la titulación que se ofrece se cataloga como privada porque se imparte en una escuela adscrita.

Como se observa en la tabla 2 las instituciones que dedican un mayor peso a la creatividad en relación con la totalidad de asignaturas de sus planes de estudio son la Universidad Autónoma de Barcelona con un 10,70\% de asignaturas dedicadas a la creatividad y la Universidad Francisco de Vitoria con un 8,75\%. Destacamos que apenas existen diferencias entre el carácter de estas instituciones ya que el porcentaje de créditos dedicados a las asignaturas de creatividad con respecto a la totalidad del plan de estudios entre instituciones de tipo público y privado difiere en un $0,62 \%$.

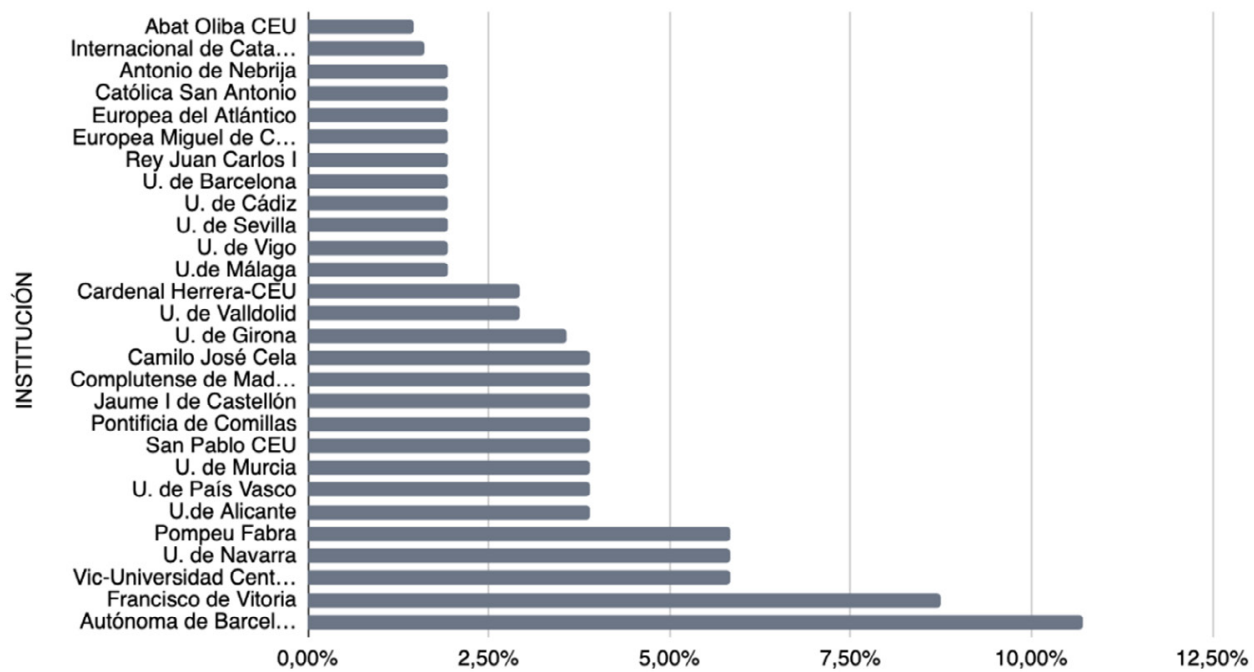

Tabla 3: Relación del número de créditos de las asignaturas sobre creatividad en relación con las instituciones universitarias españolas. Fuente: elaboración propia 
En cuanto al número de créditos correspondientes a las materias dedicadas a la creatividad, la mayoría dedican una media de 6 ECTS $(66,7 \%)$. No obstante, existen instituciones que les dedican un número menor de créditos. Un $9,8 \%$ se corresponden con asignaturas de 4 ECTS y 3 ECTS respectivamente, y un 5,9\% un total de 4,5 ECTS. Las tendencias menos comunes son materias de 12 ECTS, representando un total del 3,9\% y de 18 ECTS y 5 ECTS con un $2 \%$ respectivamente.

Con respecto a la tipología de estas asignaturas, la mayor parte $(73,08 \%)$ son asignaturas de carácter obligatorio. Este dato indica que se trata de una asignatura de carácter ineludible para la obtención del título de graduado. No obstante, un $23,08 \%$ de las asignaturas dedicadas a la creatividad se manifiestan de tipo optativo, seguidas de un 3,84\% del tipo "formación básica". El nivel en el que mayoritariamente los estudiantes cursan este tipo de materias suele ser el tercer curso $(40,4 \%)$, el segundo curso $(28,8 \%)$ y el cuarto curso $(25 \%)$. El nivel en el que se estudia la creatividad en menor media es el primer curso $(5,8 \%)$.

Por último, se han identificado varios itinerarios o menciones dentro de los planes de estudio de algunas instituciones destinados a la obtención de una especialidad relacionada con la competencia creativa. Es el caso de la Universidad Autónoma de Barcelona con la mención en "Dirección Creativa en Publicidad y Relaciones Públicas", la Universidad Pompeu Fabra con el itinerario dirigido a "Creatividad Publicitaria", o la universidad de Valladolid con un itinerario denominado “Creatividad y gestión de la creatividad de la Comunicación Publicitaria y de las Relaciones Públicas".

\section{Resultados Fase 2}

Los resultados indican que los 196 encuestados proceden de una amplia variedad de universidades entre las que destacan: Universidad de Sevilla (34 egresados), Universidad Jaume I (27 egresados), Universitat Pompeu Fabra (19 egresados), Universidad de Málaga (18 egresados), Universidad de Alicante (18 egresados), Universidad de Cádiz (12 egresados), Universidad Camilo José Cela (11 egresados), Universidad Complutense de Madrid (11 egresados), Universidad Rey Juan Carlos I (10 egresados), Cardenal Herrera-CEU (9 egresados), Universidad de Alicante (8 


\section{MONOGRÁFICO}

egresados), Vic-Universidad Central de Catalunya (7 egresados), Universidad Católica San Antonio de Murcia (5 egresados), Universidad de Girona (4 egresados), y Universidad de Valladolid (3 egresados).

Los datos indican que el $54,6 \%$ de los entrevistados se encuentra empleado por el sector publicitario. Concretamente, el $44,9 \%$ es empleado por cuenta ajena, y el 9,7\% lo hace de manera autónoma o freelance. El 45,4\% restante indica encontrarse trabajando en un sector ajeno a la publicidad $(23,5 \%)$ y el $21,9 \%$ en situación de desempleo.

En cuanto a las competencias desarrolladas durante la actividad profesional, se identifican 3 competencias básicas: la competencia digital (34,8\%), el pensamiento creativo $(22,6 \%)$ y la toma de decisiones de tipo estratégico $(17,4 \%)$. Las acciones de tipo comercial $(11,6 \%)$, la gestión económica $(8,4 \%)$ o la gestión y/o investigación documental $(4,7 \%)$, suponen el resto de las competencias más desarrolladas durante el puesto de trabajo. Otras competencias como la creación y redacción de contenido web, el diseño de producto, el diseño gráfico, la organización de eventos, la docencia, u otros, suponen el $0,5 \%$ de los resultados obtenidos revelando que las competencias digitales y estratégico-creativas son las más prácticas.

Paradójicamente, estos datos reflejan que las competencias anteriormente identificadas coinciden con aquellas que se consideran menos desarrolladas durante la formación. El 38,6\% señala que las competencias digitales han sido poco trabajadas durante el Grado, seguido de un $27,4 \%$ en lo relativo a la capacidad de decisión estratégica, y un $19,8 \%$ con respecto al pensamiento creativo. No obstante, destacan otras competencias como la organización de eventos (12,3\%), la gestión de recursos humanos $(1,7 \%) \mathrm{u}$ otros $(0,2 \%)$.

La valoración con respecto a las metodologías recibidas durante la formación en creatividad manifiesta que el 9,2\% considera que las metodologías empleadas fueron "nada adecuadas" para alcanzar los retos profesionales, el 25, 5\% "muy poco", el 42,9\% "algo", el 20,4\% considera que "mucho" y un tímido $2 \%$ que "bastante". Lo cual indica que la mayoría se inclina a realizar una valoración positiva sobre las metodologías empleadas durante la formación. 
La competencia creativa entre el alumnado de los grados en Publicidad... | 84 MONOGRÁFICO

El bloque de valoración del desarrollo de la creatividad durante la formación universitaria estimado por los encuestados revela que el 5,2\% considera que las materias dedicadas al desarrollo de la creatividad son "insuficientes", el 20,4\% "suficientes", el 59,1\% "aceptables" y un 15,3\% "excelentes".

Estos datos se relacionan con la percepción de los encuestados con respecto a la adecuación de sus estudios frente al desarrollo de sus habilidades creativas. En este caso, el 10,7\% afirma que el Grado ha contribuido "muy poco" en el desarrollo de su competencia creativa, el 17,3\% considera que "poco", el 32,1\% señala que "algo", y el 27\% y 12,8\% que "mucho" o "bastante" respectivamente.

En cuanto a los resultados obtenidos por parte de los 36 docentes, el primer bloque destinado a conocer el perfil de los encuestados señala que las universidades de procedencia de los académicos encuestados son:

Universidad de Sevilla (7 participantes), Universidad Jaume I (5 participantes), Universitat Pompeu Fabra (5 participantes), Universidad Rey Juan Carlos I (4 participantes), Universidad Complutense de Madrid (2 participantes), Universidad de Málaga (3 participantes), Universidad de Cádiz (2 participantes), Universidad de Alicante (2 participantes), Universidad Camilo José Cela (2 participantes), Vic-Universidad Central de Catalunya (1 participante), Universidad Católica San Antonio de Murcia (1 participante), Cardenal Herrera-CEU (1 participante) y Universidad de Valladolid (1 participante).

El segundo bloque, destinado a conocer la valoración de la metodología de enseñanza, se presume mayoritariamente favorable. El 25\% de los académicos señala que estas son "excelentes" para potenciar la creatividad del alumnado, el 54\% señala que son "muy buenas" y el 16,7\% considera que son "buenas". Tan solo el $4,2 \%$, esto es, un único participante, señala que son "inadecuadas".

En cuanto a la innovación de las metodologías, los docentes no parecen muy propensos al cambio en los métodos de enseñanza. El 67,3\% indica que suele cambiar sus metodologías de enseñanza-aprendizaje muy poco o nada, frente al $24 \%$ que afirma que cambia las metodologías para cada curso. El 8,7\% restante señala que rara vez realiza algún cambio. 


\section{MONOGRÁFICO}

El tercer bloque, cuyo objetivo trata de recoger la valoración de los planes de estudio en relación con las necesidades del mercado laboral, presenta un posicionamiento intermedio, aunque con una tendencia positiva. Ningún encuestado afirmó que los planes respondieran a las exigencias del mercado laboral de manera excelente, frente al 4,2\% que considera que no se adecua en absoluto. La mayoría se posiciona en una opinión intermedia $(54,2 \%)$ y el $41,7 \%$ considera que se ajustan en buena medida. No obstante, relativo a la importancia de la creatividad como un aspecto a desarrollar en estos planes, el 20,8\% considera que la creatividad ocupa un papel "poco importante", el 29,2\% considera que su peso es intermedio, el 45,8\% "importante" y el 4,2\% la considera "muy importante". Este bloque se cierra con la valoración de los docentes acerca de la importancia del desarrollo de la competencia creativa para la incorporación de los estudiantes al mercado laboral. En este caso, la mayoría $(83,3 \%)$ la considera como "muy importante", el 12,5\% “importante". Tan solo encuestado la valora como intermedia.

En cuanto a conocer la opinión de los académicos sobre el curso en el que debería desarrollarse la competencia creativa, se ha obtenido una respuesta clara: el $87,5 \%$ de los entrevistados señala que debería desarrollarse durante todos los cursos, indicando que debe ser una materia presente a lo largo de todo el plan de estudios. El resto de las posiciones indican que debería desarrollarse durante un único curso determinado. Para cada curso hubo una única votación por parte de los encuestados.

En cuanto al bloque dirigido a conocer el grado de apoyo que los docentes encuentran por parte de sus instituciones a la hora de contar con herramientas para el desarrollo de la creatividad, destaca una tendencia positiva. El 12,5\% considera que se siente "poco" apoyado por su universidad, el 33,3\% siente un apoyo "adecuado", el 41,7\% lo encuentra "muy adecuado" y el 25\% "excelente". Las respuestas también arrojaron luz acerca de la importancia que los docentes otorgan a su formación para adecuar su docencia a las demandas del perfil profesional: el 100\% de los encuestados coincide en advertir que la formación especializada y de calidad es fundamental para el correcto desarrollo de su actividad docente. 
La competencia creativa entre el alumnado de los grados en Publicidad... | 86

MONOGRÁFICO

\section{Fase 3}

El desarrollo de las entrevistas a los expertos fue fluido y colaborativo. En términos generales, la percepción de los consultados sobre la formación universitaria presenta cierto déficit en la formación en creatividad. Según los expertos; "no hay suficiente nivel para lo que exige el mercado laboral, no vienen trabajados en creatividad" (E4) y es que, según el experto 3 (E3), esta formación previa se nota "en muy pocas ocasiones". De hecho, aunque "sí que tienen conocimientos sobre publicidad, les falta dar rienda suelta a las opciones que les da la creatividad publicitaria en todas sus formas" (E7). Concretamente, el E6 afirmaba que la formación en creatividad "sería muy positivo que se impartiera de manera continuada durante todos los cursos" (E6) ya que como apoyaba el experto 8: "A la hora de la verdad es lo más práctico" (E8).

Casi todos los expertos coinciden en que los graduados no salen con una preparación suficiente en el ámbito creativo. El experto 1 reconocía que "la persona que sale con una preparación aceptable es la que ha tomado tiempo para complementar la formación universitaria con una formación autodidacta en paralelo" (E1) o el experto 5 quien manifestaba que "de las universidades creo que no traen la formación suficiente, aunque vengan medianamente formados" (E5). De hecho, la tónica general de las aportaciones se posiciona hacia la poca adecuación a la realidad: "Creo que les falta profundizar más en este terreno. Tienen recursos y herramientas, pero dista mucho de lo que se pide en agencias" (E4). Incluso cuando en algún caso se posicionan claramente a favor de la formación universitaria, nos encontramos con una respuesta negativa: "Soy súper defensora de la universidad y pública, pero sin haber pasado por una escuela de creatividad no hubiese podido trabajar nunca en una agencia de primera línea" (E8).

A la hora de contratar a un joven en prácticas, los expertos coinciden en la importancia de poder valorar trabajos previos de los estudiantes: "valoro mucho las ideas que plantean en sus carpetas para resolver los problemas planteados. Aunque sean campañas ficticias" (E3). Los expertos E1 y E6 señalan la importancia del portfolio creativo cuando manifiestan que "prácticamente el $100 \%$ de los alumnos que aplican para el departamento creativo adjuntan un portfolio. De hecho, sin portfolio esa candidatura es descartada" (E6). 


\section{MONOGRÁFICO}

Las principales cualidades que los estudiantes y recién egresados deben mejorar para incorporarse al sector de la publicidad fueron discutidas por los participantes, quienes concretaban que es necesario que los egresados trabajen "su sello personal y perfilen sus capacidades creativas para conseguir un estilo que les haga diferenciarse" (E3). "Tener una visión global de cómo transformar una idea brillante en distintas ejecuciones" (E7). Todo ello, al final, se resume en la aportación del E8: "Conceptualización y referencias. Aprender a pensar".

Atendiendo a los roles profesionales con mayor proyección y empleabilidad en el sector, algunos expertos apuestan por perfiles relacionados con la innovación (E1). Para otros "los perfiles creativos tradicionales llegan más lejos que los perfiles estratégicos o de cuentas" (E2). Y, entre ambas posturas, encontramos diversas opiniones: como que "los creativos digitales destacan sobre los demás" (E3), o quienes apuestan por un rol muy concreto: “La dirección de arte" (E4). Los perfiles híbridos son citados por los E5 y E6.

Para finalizar, cuando planteamos cuáles son las principales competencias que debe tener un egresado en Publicidad los expertos consensuan ciertas características como: la paciencia, la motivación, la curiosidad, el saber expresarse, la proactividad, no tener prejuicios $\mathrm{y}$, sobre todo, ser valientes en plantear las ideas y llevarlas al mundo real (E1-E8).

\section{Discusión y conclusiones}

Los resultados extraídos de las distintas fases metodológicas ofrecen un interesante punto de partida para analizar y poner en común las actuales demandas laborales vinculadas al ejercicio de la creatividad publicitaria en España y los planes de estudios del Grado en Publicidad y Relaciones Públicas. Atendiendo al primer objetivo, si bien el análisis realizado manifiesta que el carácter obligatorio de las asignaturas creativas demuestra la forzosa necesidad de cursar este tipo de materias para obtener la titulación, el número de créditos totales en relación con la totalidad del Grado revela cierta insuficiencia de materias creativas en relación a otros contenidos. Los expertos coinciden en que los graduados no presentan una formación suficiente con respecto a las demandas del mercado. Algo de lo que se hacen eco los egresados 
cuando, a tenor de los comentarios ofrecidos, señalan una falta del desarrollo de sus habilidades creativas percibidas durante el Grado. En opinión de los académicos, estos parecen encontrarse más alejados de la realidad profesional en tanto que sus respuestas indican ciertas diferencias a la hora de reconocer un consenso sobre la importancia de la creatividad en los planes de estudio.

Algunos de los factores que podrían explicar estas tendencias son, por un lado, el exceso de integración de competencias digitales en la renovación del Grado. La respuesta ante la tendencia actual hacia la digitalización del trabajo ha sido una de las preocupaciones fundamentales a la hora de actualizar los planes de estudio obviando competencias tradicionales del Grado como la creatividad. Siguiendo la opinión de los egresados, si bien las destrezas digitales son muy demandas en el sector profesional, las habilidades creativas son también reconocidas como capacidades fundamentales en su trabajo diario.

Por otro lado, las exigencias profesionales sufridas por los docentes podrían ofrecer una respuesta a esta insuficiencia en el desarrollo del trabajo creativo. Los académicos reconocen sentir cierta comodidad a la hora de diseñar sus modelos de enseñanza. No obstante, reconocen que el cambio en las metodologías es un proceso lento. Es importante destacar que el papel del profesor universitario actualmente se ve verdaderamente afectado por la disparidad de exigencias tanto docentes y de investigación conduciendo en ocasiones al colapso profesional. No obstante, la mayoría de los egresados se inclina a realizar una valoración positiva sobre las metodologías empleadas en clase por parte de los docentes.

En cuanto a los objetivos acerca de examinar el ajuste de los planes de estudio a los perfiles creativos que se demandan en el campo profesional (0.1.1) y su posterior evaluación en cuanto a su adecuación en la formación recibida con respecto a su desempeño profesional (0.1.2), los expertos señalan lo que denominan una insuficiencia en la preparación del alumnado. La tendencia observada entre los profesionales es que, si bien la formación parece adecuada $y$, de hecho, se identifican ciertas aptitudes entre los jóvenes profesionales, es cierto que apuntan hacia una necesidad de complementar la formación. Como ya manifestaron otros estudios como los de Farfán y Corredor (2010) o Castell-Martínez (2020) sobre las carreras de comunicación en 


\section{MONOGRÁFICO}

general, para el caso que nos ocupa la formación recibida no parece del todo adecuada para responder a tales demandas. La realidad profesional exige una mayor dedicación al trabajo creativo que parece no reconocida en los planes de estudio. De hecho, el examen de estos manifiesta una baja proliferación de itinerarios especializados, así como un desequilibrio de la competencia creativa en el reparto de los cursos. Es, por tanto, necesario ofrecer una oferta formativa que responda a las necesidades creativas profesionales en el Grado en Publicidad y Relaciones Públicas.

No obstante, es preciso señalar que los resultados de las encuestas realizadas a los egresados demuestran cierta eficiencia a la hora de la incorporación al mundo laboral ya que apenas existen desempleados en el sector. Teniendo en cuenta lo anteriormente expuesto, podríamos afirmar la hipótesis de que, en efecto, las actuales demandas laborales vinculadas al ejercicio de la creatividad publicitaria en España requieren de una transformación de los planes de estudio universitarios del Grado en Publicidad y Relaciones Públicas. En este sentido, si entre nuestros objetivos no se encuentra conocer de qué modo deben realizarse esas transformaciones, sí ofrece un valioso punto de partida para reconocer algunas de las debilidades que actualmente $\mathrm{y}$, atendiendo al mercado laboral, deben de mejorar para dar respuesta a la realidad profesional. Cuestiones como el refuerzo creativo de tipo práctico a través de estrategias como el diseño de portfolios creativos, el aumento de asignaturas creativas a lo largo de todo el Grado o la atención a las demandas del profesorado para facilitar un método más vinculado a la profesión, son algunas de las cuestiones que podrían valorarse para futuras investigaciones.

\section{Referencias}

Agüero Pérez, M.M., López Alonso, E., López Fraile, L.A., y Moreno López, B. (2019). Implicación de los stakeholders en la creación del Grado en Publicidad en la Universidad Europea. Imbricación del sector profesional en el entorno académico. Revista Latina de Comunicación Social, 74, 50-72. doi: 10.4185/ RLCS-2019-1321

Alameda-García, D., Fernández-Blanco, E., y Benavides-Delgado, J. (2013). El sector publicitario en su triple vertiente económica, estructural y cultural, Palabra Clave, 16(1), 182-214. URL: https://bit.ly/3eLZ3pY 
La competencia creativa entre el alumnado de los grados en Publicidad... | 90 MONOGRÁFICO

Alonso, L.E., Fernández Rodríguez, C.J., y Nyssen, J.M. (2009). El debate sobre las competencias. Una investigación cualitativa en torno a la educación superior y el mercado de trabajo en España. Madrid: ANECA.

ANECA. (2005). Libro Blanco. Títulos de grado en Comunicación. Madrid: Agencia Nacional de Evaluación de la Calidad y Acreditación. URL: https://bit. ly/2MK8IkB

Bain, K. (2004). What the Best College Teachers Do? Cambridge/Londres: Harvard University Press.

Castelló-Martínez, A. (2020). Las asignaturas de creatividad y estrategia en los Grados en Comunicación en España. Revista Latina de Comunicación Social, (77), 143-178. https://www.doi.org/10.4185/RLCS-2020-1453

Costa, J. (2015). Creatividad, invención e innovación. Revista Aportes de la Comunicación y la Cultura, (19), 27-30. URL: https://bit.ly/2XV7CI5

Cuenca, J., Compte, M., Matilla, K., y Hernández, S. (2017). Un análisis de los másteres universitarios españoles especializados en Relaciones Públicas y Comunicación Corporativa curso 2016-2017 desde la perspectiva de las Relaciones Públicas. Obra Digital, (13), 37-57. doi: https://doi.org/10.25029/ od.2017.156.13

Farfán, J. y Corredor, P. (2010). Demandas y formación: Nuevos perfiles profesionales para la publicidad en España. Pensar en la publicidad, 4(1), 97-166. URL: https://bit.ly/2zWOGT7

Fueyo, A., Rodríguez-Hoyos, C. y Linares, C. (2015). La innovación docente de la formación de los profesionales de la educación: el papel de la educación mediática. En Ferrés, J. \& Masanet, MJ. La educación mediática en la universidad española, pp. 31-51. Barcelona: Gedisa.

González-Oñate, Cristina (Coord). (2019). El negocio publicitario en la sociedad digital de la Comunidad Valenciana. Barcelona: UOC

Imbernón, F. y Guerrero, C. (2018). ¿Existe en la universidad una profesionalización docente? Revista de Educación a Distancia, (56)11, 2-12.doi: http://dx.doi. $\mathrm{org} / 10.6018 / \mathrm{red} / 56 / 11$

Krippendorf, K. (2004). Análisis de contenido: Una introducción a su metodología. Washington D.C: SAGE.

Linstone, H. y Turoff, M. (2002). The delphi method. Reading: Addison-Wesley. 
López-Font, L. (2005). Agencia de publicidad: La reinvención constante. Questiones Publicitarias, 1(10), 99-118.doi: https://bit.ly/3dwF0An

Martínez, B., Martínez, I., Alonso, I., y Geruzaga, M. (2013). El aprendizajeservicio, una oportunidad para avanzar en la innovación educativa dentro de la universidad del País Vasco. Técnicas pedagógicas, 21, 99-117. URL: https:// bit.ly/2BtNTIO

Martínez Olmo, F. (2002). El cuestionario: un instrumento para la investigación en las ciencias sociales. Laertes: Barcelona.

Matilla, K., Cuenca-Fontbona, J., y Compte-Pujol, M. (2018). Un análisis de las asignaturas sobre Comunicación y Relaciones Públicas en los másters universitarios españoles tipo MBA (curso 2016-2017). adComunica. Revista Científica de Estrategias, Tendencias e Innovación en Comunicación, (16), 221240. URL:https://bit.ly/36WiSbb

McKinsey Global Institute. (2018). Skill Shift. Automation and the future of the Workforce. Recuperado de https://mck.co/2U4TWsK

Observatorio de la Publicidad en España. (2019). Informe Observatorio de la Publicidad en España Recuperado de https://bit.ly/3dtAU7p

Peinado Miguel, F., y Fernández Sande, M. (2011). Reflexión sobre la motivación de los alumnos de Grado en la elección de estudios de comunicación en las universidades de Madrid. Estudios sobre el mensaje periodístico, 17(2), 383400. URL: https://bit.ly/3gQRrEb

Robinson, K. (2009). El elemento. Descubrir tu pasión lo cambia todo. Editorial Debols!llo Clave: Madrid.

Román-San-Miguel, A. (2014). Nuevos roles del profesorado universitario en Periodismo en la era de las redes sociales. En Fombona, J. y Caldevilla, D. (coord.) Nuevas formulaciones de los contenidos docentes, pp. 531-538. Madrid: McGraw Hill.

Ruíz, C. y Martín, C. (2005). Innovación docente en la Universidad en el marco del EEES. Educatio Siglo XXI, 23, 171-189. URL: https://bit.ly/306jc50

Sáenz, 0. (1990). Actitudes de los profesores ante la integración del niño discapacitado en la escuela ordinaria: una visión desde la literatura científica. Revista interuniversitaria de formación del profesorado, (8), 135-150.

Sánchez Pozo, A. S. (2008). Elementos clave en el diseño de módulos y titulaciones. Revista de Investigación en Educación, 5(1), 41-48.URL: https://bit.ly/2Xr4g03 
Selva Ruíz, D. y Domínguez Liñán, R. (2018). Las técnicas de generación de ideas: revisión y análisis de su uso en las agencias publicitarias española. Área Abierta. Revista de comunicación audiovisual y publicitaria, 18(3), 371-387. http://dx.doi.org/10.5209/ARAB.56763

Scopen. (2019). Informe Agency Scope 2018/2019. Recuperado de https://bit. ly/2XXocHq

Solanas, I. y Sabaté, J. (2011). Dirección de cuentas: gestión y planificación de cuentas en publicidad. Barcelona: UOC.

Torres, F. (2008). La evaluación y el enfoque de competencias: tensiones, limitaciones y oportunidades para la innovación docente en la universidad. Revista escuela de administración de negocios, (63), 91-105. URL: https://bit. ly/2Xqm8Zg

Turnbull, S. y Wheeler, C. (2016). Exploring advertiser's expectations of advertising agency services. Journal of Marketing Communications, 6(22), 587-601.doi: https://doi.org/10.1080/13527266.2014.920902

Vázquez Gestal, M. y Fernández Souto, A.B. (2012). Las necesidades formativas de los nuevos comunicadores según los profesionales y su reflejo en los Grados de Comunicación. Estudios sobre el mensaje periodístico. Número especial Noviembre (18), 889-897.doi: https://doi.org/10.5209/rev ESMP.2012.v18.40967

Vázquez Gestal, M. (2011). Desarrollo de la creatividad publicitaria. Pasado y presente. Correspondencias \& Análisis,1, 175-190. https://bit.ly/2XxkjKp

Villamizar, G. (2012). La creatividad desde la perspectiva de estudiantes universitarios. Revista Iberoamericana sobre Calidad, Eficacia y Cambio en Educación, 2(10), 212-237. https://bit.ly/2U3z8C2

\section{(c) $\underset{\mathrm{EY}}{(-)}$}

Este obra está bajo una licencia de Creative Commons Reconocimiento 4.0 Internacional. 